\title{
The Role of Diffusion MRI in Rectum Cancer Staging and Evaluation of Neoadjuvan Treatment Efficiency
}

\author{
Mustafa YILDIRIM' ${ }^{1}$ Mustafa KOC ${ }^{2}$ \\ ${ }^{1}$ Elazig Fethi Sekin City Hospital, Department of Radiology \\ ${ }^{2}$ Firat University, Faculty of Medicine, Department of Radiology, Elazig, TURKEY
}

\begin{abstract}
The correct staging of rectal cancer is very important for treatment. This study aims to show the contribution of diffusion-weighted imaging (DWI) to staging, to predict tumor differentiation and neoadjuvant chemoradiotherapy response using DWI. The study consisted of 36 patients and 22 control groups. 12 patients who received neoadjuvant therapy were evaluated before and after treatment. Magnetic Resonance Imaging (MRI) and DWI were performed to all patients and apparent diffusion coefficient (ADC) maps were obtained. The findings were compared with histopathological results. T staging accuracy was $72.2 \%$ on MRI. N staging accuracy rate was $75 \%$ on the T2 sequence and $72.2 \%$ on DWI. Tumoral rectal ADC values were significantly decreased compared to the normal rectal wall $(p<0.001)$. Mean T3 and T4 (extramural) ADC values were significantly decreased compared to the T2 stage (intramural) $A D C$ values $(p<0.001)$. ADC and relative ADC (lymph node / primary tumor $A D C$ ) values of the metastatic lymph nodes were significantly decreased compared to benign lymph nodes ( $p<0.001$ ). According to the ADC cut point, $N$ staging accuracy was found to be $83 \%$. The ADC values of the low differentiated group were significantly decreased compared to the moderately and well-differentiated group ( $p<0.011)$. In the control MRI of patients receiving neoadjuvant therapy, the ADC increase in the group that responded well to the treatment was significantly higher than the group with partial response $(p<0.004)$. As a result, DWI and ADC are useful for preoperative rectum cancer evaluation.
\end{abstract}

Keywords: Rectum cancer, Staging, Diffusion MRI

\section{INTRODUCTION}

Colorectal cancers are the third most common cancer in men and the second in women. ${ }^{1}$ Colorectal cancers account for about $16 \%$ of total cancer cases in the world, with $14 \%$ in cancer-related deaths. ${ }^{2}$ Rectal cancer has a worse prognosis compared to other colon tumors with the rate of metastasis and local recurrence. ${ }^{3}$

The prognosis of rectum cancer is directly related to the stage. Factors determining prognosis; depend on the mural (T1-T2), extramural (T3-T4) spread of the tumor, mesorectal fascia invasion, lymph node spread, and the presence of distant metastasis . ${ }^{4}$
Rectal cancer treatment is determined by local staging. Early-stage tumors (T1, T2, N0) are treated surgically only. Patients with locally advanced rectal cancer (T3, T4, N+) receive neoadjuvant chemoradiotherapy before surgery. Correct tumor staging and appropriate neoadjuvant therapy reduce tumor stage and dramatically reduce local recurrence. ${ }^{5}$ In addition, neoadjuvant therapy allows sphincter-sparing surgery and increases prognosis positively. ${ }^{6} \mathrm{MRI}$ is the best modality in preoperative rectal cancer staging. It also plays a vital role in post-neoadjuvant control assessment and for treatment planning. ${ }^{7}$ 
DWI is a non-invasive method based on the diffusion of water molecules, showing the biological properties of tissue. ${ }^{8}$ ADC (Apparent Diffusion Coefficient) map is created with the relative difference in tissue diffusion and the ADC map allows measurement of ADC values. This quantitative analysis has been shown to be useful for evaluating tumors and distinguishing benign-malignant lesions. ${ }^{8}$ Low ADC values reflect cellular density. Recent studies have shown that the ADC value reflects tumor aggressiveness. ${ }^{9}$ In addition, intratumoral changes induced by chemoradiotherapy can be calculated. ${ }^{10}$

The aim of this study is to show the contribution of diffusion MRI to staging in patients with rectal cancer, to predict neoadjuvant treatment response and tumor differentiation with diffusion MRI.

\section{PATIENTS AND METHODS}

This prospective study was approved by the Ethics Committee. All patients signed a consent form. Between 2015 and 2018, 36 cases who were diagnosed with rectum adenocarcinoma as a result of the colonoscopic biopsy were included in the study. The age range of the cases was 39-87. Only surgical treatment was applied to 24 cases within one month after MRI. Pre-op MRI findings and post-op histopathological findings were compared. 12 patients received preop neoadjuvant therapy and MR images of 12 patients were evaluated before and after neoadjuvant therapy. After the neoadjuvant treatment, MR findings and post-op histopathological findings were compared. Twenty-two patients with normal colonoscopic examination were accepted as the control group. These patients had thick rectum wall on MR

\section{MRI Protocol}

1.5 Tesla MRI with 32 channels and the superficial coil was used. Images were obtained in the supine position. In the lower abdomen MRI examination; T2 axial, T2 coronal and sagittal plans, axial T1 and axial T1 dynamic sections were taken.

Spin echo single-shot echo-planar (ss-EPI) sequence was used as DWI. The values of b0, b100 and b600 were used on DWI. ADC maps of all patients were created. In T2 sequence, matrix size was taken as $320 \times 224$, NEX 2.0, FOV $24 \times 24 \mathrm{~cm}$, section thickness $5 \mathrm{~mm}$, inter-sectional space 0.5 mm, TR 7008 ms, TE 109 ms. In DAG sequence, matrix 80 x 128, NEX 4.0, FOV 40 x $40 \mathrm{~cm}$, section thickness $4 \mathrm{~mm}$, TR 5000 and TE 61.9 were taken.

\section{Evaluation of MR Images}

MR images were evaluated at the workstation (PHILIPS workstations) without knowing histopathological findings. $\mathrm{T}, \mathrm{N}$, and $\mathrm{M}$ staging of the tumor was performed according to the AJCC (American Joint Committee on Cancer) TNM staging system with 1.5 Tesla MRI. ${ }^{11}$ Staging was done according to the T2 sequence. T staging (mT) of MRI was compared with histopathological T staging (pT). Then, according to histopathological results, ADC measurements were made from T4, T3, T2 stage tumor walls with b600 values of DWI. ADC measurements were made with $5 \mathrm{~mm}^{2}$ ROI from 3 focuses with the lowest signal. The average of these three ADC values was calculated. 22 control cases with normal rectum walls were also taken ADC measurements. In addition, The mean $\mathrm{ADC}$ values of primary tumors with and without lymph node metastasis were compared.

Lymph nodes that bigger than $5 \mathrm{~mm}$, with heterogeneous signal and irregular contours on the T2 weighted image, were accepted pathological. DWI was used as the other method. DWI hyperintense and ADC hypointense, over $5 \mathrm{~mm}$ lymph nodes were accepted pathological. According to these two different parameters, if no lymph node was detected, the nodal staging was accepted as N0. 1-3 pathological lymph nodes were evaluated as N1. 4 or more pathological-looking lymph nodes were evaluated as N2. Histopathological N staging was compared with MRI findings. Then, according to the histopathological results, ADC values were measured from the lymph nodes of patients with N0, N1-2. Measurements were made from lymph nodes of at least $5 \mathrm{~mm}$ in size. b600 values were used. Relative ADC values of lymph nodes (Lymph Node ADC / Rectum ADC) were calculated and compared. Primary tumor ADC values were compared histopathologically with tumor differentiation grades. In addition, primary tumor ADC 
values and tumor volume obtained from pathological specimens were compared.

In patients receiving neoadjuvant therapy, ADC values after and before treatment were subtracted from each other. $\triangle \mathrm{ADC}$ (ADC value after neoadjuvant therapy - ADC value before treatment) was calculated and compared with histopathological tumor regression grading. Histopathological tumor regression grading (TRG) was scored according to the Mandard's system. ${ }^{12}$ TRG 1: (complete response), no residual cancer and fibrosis along the wall. TRG 2: rare cancer cells scattered throughout fibrosis. TRG3: increased cancer cells, but still fibrosis dominant TRG4: (minimal response), dominant residual cancer, partial fibrosis. TRG5: (unresponsive), no regression change.

\section{Neoadjuvant Radiotherapy and Chemotherapy}

Radiotherapy treatment volume was determined to cover the proximal and distal 3-5 cm distance of the tumor. Perirectal, obturator, internal iliac, external iliac, and presacral lymph nodes were included. Radiotherapy with 18 MV photon energy was applied to this volume with a linear accelerator device in all patients. Pelvic radiation at a total dose of $45 \mathrm{~Gy}$ in 25 fractions was applied for 5 days each week for 5 weeks. $800 \mathrm{mg} / \mathrm{m}^{2}$ capecitabine was administered as a chemotherapeutic agent with radiotherapy.

\section{Statistical Analysis}

Numerical measurements was summarized as median and minimum - maximum. The Kolmogorov Smirnov test was used to test whether numerical measurements provide the normal distribution assumption. The Mann Whitney U test was used to compare numerical measurements that did not show normally distribution between the two groups, while the Kruskal Wallis test was used for general comparison between more than two groups. Spearman test was applied for the correlation test. ROC Analysis was performed to determine the appropriate cutting points for the ADC variable. IBM SPSS Statistics 21.0 (SPSS Inc, Chicago, IL, USA) program was used for data analysis. Statistical significance level was taken as $\mathrm{p}<0.05$ in all tests.

\section{RESULTS}

Histopathologically, 12 (33\%) patients were defined as T2, $12(33 \%)$ patients as T3, and $12(33 \%)$ patients as T4. According to the T2 sequence, 18 of 24 patients who did not receive neoadjuvant therapy were staged correctly (75\%). Eight of 12 patients who received neoadjuvant therapy were staged correctly (66\%). Totally 26 patients $(72.2 \%)$ were staged correctly in T staging. 3 cases $(8,3 \%)$ were staged upper and 7 cases $(19,4 \%)$ were staged lower. 9 of the $12 \mathrm{~T} 2$ stage patients $(75 \%)$, were correctly staged, 3 of them (25\%) were staged upper. 9 of 12 T3 stage patients (75\%) were correctly staged, 3 of them (25\%) were staged lower (T2). 8 of 12 T4 stage patients $(66,6 \%)$ were correctly staged and 4 of them (33.3\%) were staged as substage (T3) (Table 1a).

Histopathologically, 18 (50\%) patients were N0, $9(25 \%)$ patients were N1, 9 patients $(25 \%)$ were N2. According to the T2 sequence, 19 of 24 patients who did not receive neoadjuvant therapy were staged correctly (79\%). 8 of 12 patients who received neoadjuvant therapy were staged correctly (66\%). Totally 27 of 36 patients $(75 \%)$ were staged correctly in $\mathrm{N}$ staging. 6 patients $(16.6 \%)$ were staged upper, 3 patients $(8.3 \%)$ were staged lower. According to the DWI sequence, 18 of 24 patients who did not receive neoadjuvant therapy were staged correctly (75\%). 8 of 12 patients who received neoadjuvant therapy were staged correctly $(66 \%)$. According to the DWI sequence, a total of 26 patients $(72.2 \%)$ were staged correctly. 8 patients $(22.2 \%)$ were staged upper 2 patients $(5.5 \%)$ were sub-staged (Table 1b).

\section{Rectum ADC Values}

The result of statistical analysis for rectum ADC values; when compared with the control group, a statistically significant decrease was observed in the T2 (p<0.001), T3 ( $<<0.001)$ and T4 (p<0.001) groups. Compared to the $\mathrm{T} 2$ group, a statistically significant decrease was observed in the T3 $(\mathrm{p}<$ $0.001)$, and T4 $(\mathrm{p}<0.001)$ groups. However, there was no statistically significant difference between the T3 and T4 groups for the rectum ADC values $(p=0.297)$ (Table 1c). In separating rectal cancer and normal rectum wall, the receiver operating 
International Journal of Hematology and Oncology

Table 1. Study data and statistical results

a. Comparison of MRI and histopathology findings for T staging

\begin{tabular}{lllll|} 
& pT1 & pT2 & pT3 & pT4 \\
\hline mT1 & 0 & 0 & 0 & 0 \\
mT2 & 0 & 9 & 3 & 0 \\
mT3 & 0 & 3 & 9 & 0 \\
mT4 & 0 & 0 & 4 & 8
\end{tabular}

b. $N$ staging according to $T 2$ and $D A G$

\begin{tabular}{|llll}
\hline & Correct staging & Upper staging & Lower staging \\
\hline T2 & $27(75 \%)$ & $6(16.6 \%)$ & $3(8.3 \%)$ \\
DAG & $26(72.2 \%)$ & $8(22.2 \%)$ & $2(5.5 \%)$
\end{tabular}

c. Median (min-max) values of rectum ADC measurements

Rectum ADC Values Median (min-max)

CONTROL $1.93(1.55-2.35) \times 10^{-3}$

$\mathrm{T} 2$

T3

$1.38(1.13-1.70)^{\mathrm{a}} \times 10^{-3}$

$1.03(0.76-1.29)^{\mathrm{ab}} \times 10^{-3}$

$0.95(0.76-1.06)^{\mathrm{ab}} \times 10^{-3}$

$<0.001^{*}$

${ }^{a}$ Compared with the control group. ${ }^{b}$ Compared with the T2 group. $(p<0.05)$

d. Rectal cancer ADC values with and without lymph node metastasis

Rectal cancer ADC Values Median (min-max)

N1-2 Rectal cancer

$1.13(0.76-1.70) \times 10^{-3}$

No Rectal cancer

$p$

$1.10(0.77-1.46) \times 10^{-3}$

$=0.733$

characteristic (ROC) curve was done to evaluate the diagnostic capability of the ADC value. Area under the curve (AUC) was $0.996(0.930-1.000, \mathrm{p}<$ $0.001)$. The cut point obtained depending on the Youden index was found $1.52 \times 10^{-3} \mathrm{~mm}^{2} / \mathrm{sec}$. Sensitivity was $94 \%$ and specificity was $100 \%$ at this cut-point (Figure 1).

There was no statistically significant difference in ADC values of rectum cancers with and without lymph node metastasis. However, the mean ADC value of the group that did not perform lymph node metastasis was minimally higher $(\mathrm{p}=0.733)$ (Table $1 \mathrm{~d})$.

\section{Lymph Nodes ADC Values}

Histopathologically 18 N0 patients had 18 lymph nodes that $5 \mathrm{~mm}$ and above. ADC measurement was performed from these benign lymph nodes. As a result of histopathology, 18 patients with N1-2 had 62 metastatic lymph nodes. ADC was meas- ured from 36 lymph nodes $5 \mathrm{~mm}$ and above, most likely to be metastatic. When compared with benign lymph nodes, a statistically significant decrease was observed in the ADC values of metastatic lymph nodes $(\mathrm{p}<0.001)$ (Table $2 \mathrm{a})$. In ROC analysis of ADC values of lymph nodes, AUC was $0,928$ (0.824 - 0.981, $\mathrm{p}<0.001)$. The cut point obtained depending on the Youden index was found $1.33 \times 10^{-3} \mathrm{~mm}^{2} / \mathrm{sec}$. Sensitivity was $75 \%$ and specificity was $100 \%$ at this cut-point (Figure 2a). In lymph node staging with $1.33 \times 10-3 \mathrm{~mm}^{2} / \mathrm{sec}$ cut off value, 18 of $18 \mathrm{~N} 0$ patients were staged correctly. 12 of 18 N1-2 patients were staged correctly. 6 of them were staged lower. The accuracy rate was $83.3 \%$.

Compared with the relative ADC values of benign lymph node, a statistically significant decrease in metastatic lymph node relative ADC values was observed ( $\mathrm{p}<0.001$ ) (Table 2b). According to ROC analysis of relative ADC values of lymph nodes, 


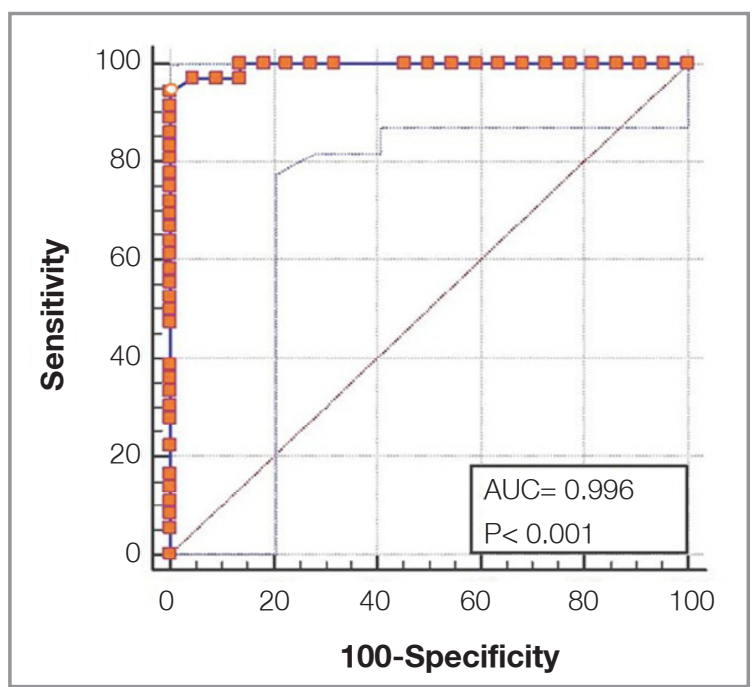

Figure 1. $R O C$ analysis of rectum $A D C$ values

AUC was 0.933 (0.823 - 0.985, $\mathrm{p}<0.001)$. The cut point obtained depending on the Youden index was found $1.47 \times 10^{-3} \mathrm{~mm}^{2} / \mathrm{sec}$. Sensitivity is $90.62 \%$ and specificity is $88.24 \%$ at this cut-point (Figure 2b). In lymph node staging with $1.47 \times 10^{-3} \mathrm{~mm}^{2} /$ sec cut off value, 15 of $18 \mathrm{~N} 0$ patients were staged correctly. 3 of them were staged upper. 15 of 18 N1-2 patients were staged correctly. 3 of them were staged lower. The accuracy rate was $83.3 \%$.

\section{ADC Values According to Tumor Differentiation Degree}

Histopathologically, 4 (11\%) patients were poorly differentiated, 26 (72\%) patients were moderately differentiated and $6(17 \%)$ patients were well-differentiated. When compared with the poorly differentiated group, a statistically significant increase in $\mathrm{ADC}$ values in the moderately differentiated $(\mathrm{p}<$ $0.011)$ and well-differentiated $(p<0.010)$ group was observed. However, there was no statistically significant difference between moderately differentiated and well differentiated groups $(p=0.069)$ (Table 2c).

\section{Comparison of $\triangle \mathrm{ADC}$ with Pathological Respons}

\begin{tabular}{|c|c|}
\hline \multicolumn{2}{|l|}{ a. Lymph Nodes ADC Values } \\
\hline & Lymph Nodes ADC Values Median (min-max) \\
\hline Benign lymph nodes & $2.11(1.34-3.25) \times 10^{-3}$ \\
\hline Metastatic lymph nodes & $1.17(0.74-2.00) \times 10^{-3}$ \\
\hline$p$ & $<0.001^{\star}$ \\
\hline \multicolumn{2}{|c|}{ b. Lymph node ADC/ Rectum ADC values } \\
\hline & Relative ADC Values Median (min-maks) \\
\hline Benign Relative ADC Values & $2.00(1.21-3.52) \times 10^{-3}$ \\
\hline Metastatic Relative ADC Values & $1.11(0.60-1.97) \times 10^{-3}$ \\
\hline$p$ & $<0.001^{*}$ \\
\hline \multicolumn{2}{|c|}{ c. ADC Values According to Tumor Differentiation Degree } \\
\hline & Rectal cancer ADC Values Median (min-max) \\
\hline Poorly differentiated & $0.86(0.76-0.96) \times 10^{-3}$ \\
\hline Moderately differentiated & $1.12(0.76-1.70)^{\mathrm{a}} \times 10^{-3}$ \\
\hline Well differentiated & $1.26(1.08-1.37)^{\mathrm{a}} \times 10^{-3}$ \\
\hline$p$ & $0.007^{\star}$ \\
\hline \multicolumn{2}{|c|}{${ }^{a}$ Compared with poorly differentiated group } \\
\hline \multicolumn{2}{|c|}{ d. Comparison of $\triangle \mathrm{ADC}$ with Pathological Response } \\
\hline & $\triangle \mathrm{ADC}$ Median (min-max) \\
\hline TRG 1-2 & $0.67(0.40-0.90) \times 10^{-3}$ \\
\hline TRG 3-4 & $0.28(0.20-0.35) \times 10^{-3}$ \\
\hline $\mathrm{p}$ & $0.004^{*}$ \\
\hline
\end{tabular}




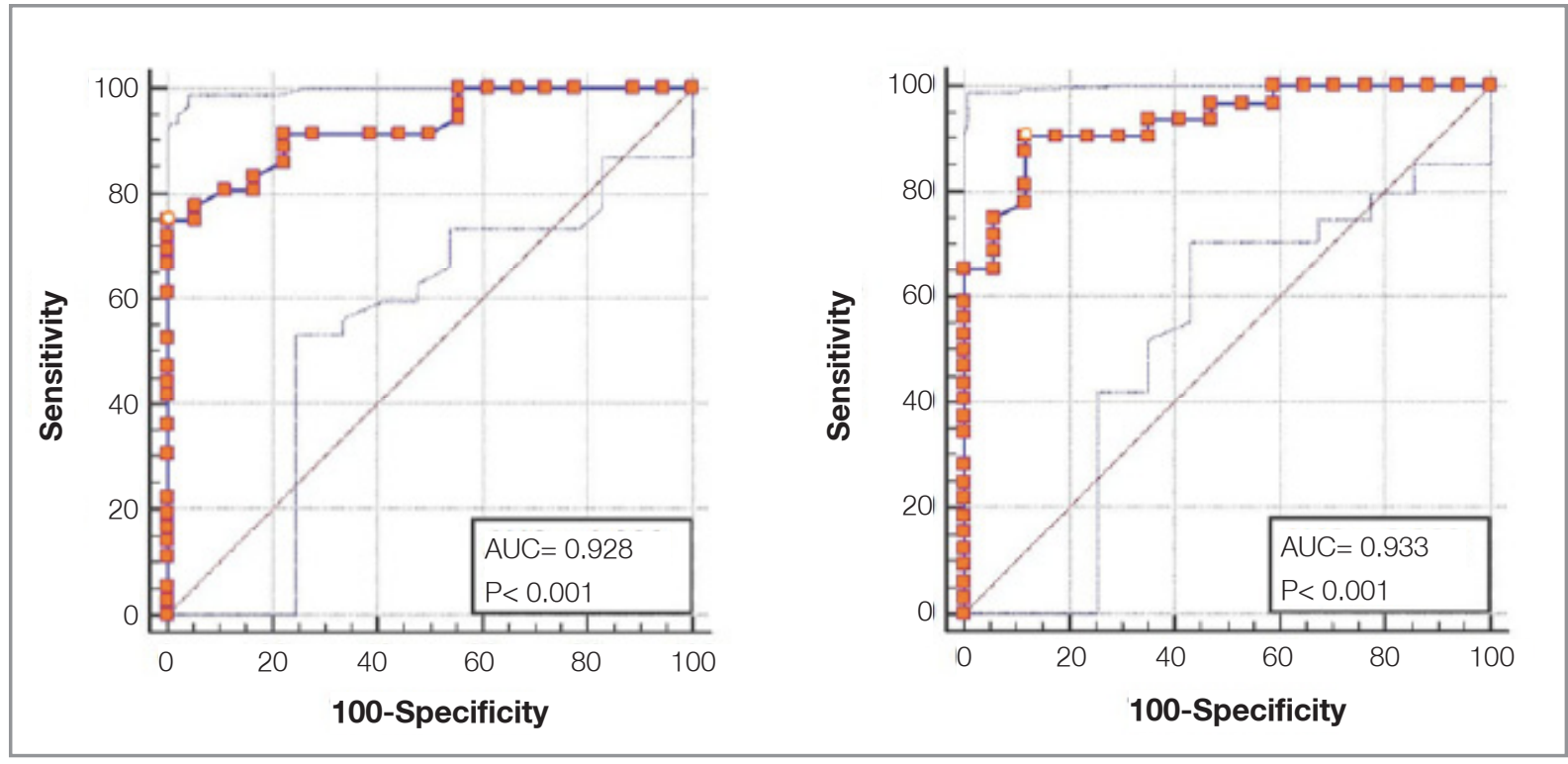

Figure $\mathbf{2 a}$ and $\mathbf{2 b}$. ROC analysis of benign-metastatic lymph nodes ADC and relative ADC values

12 patients received neoadjuvant therapy. According to the Mandard regression analysis, 4 of them responded well to treatment (grades 1 and 2) and 8 of them responded partially to treatment. (grade $3,4)$. Compared with the partial response, in the group with a good answer, there was a statistically significant increase in $\triangle \mathrm{ADC}$ values $(\mathrm{p}=0.004)$ (Table 2d).

There was a weak and negative correlation between histopathologic tumor volume and ADC values, but this correlation was statistically insignificant $(r=-0.076 ; p=0.66)$.

\section{DISCUSSION}

Colorectal cancers are one of the most common cancers worldwide. ${ }^{13}$ It is one of the major causes of cancer-related deaths and the rectal cancer mortality rate is $4-10 / 10000$ every year. ${ }^{14}$ Rectal cancer prognosis depends on the age of the patient, depth of invasion, lymph node metastasis, circumferential resection margin and extramural vascular invasion. ${ }^{15}$ The 5-year rectal cancer survey rate was found $66.6 \%, 88.2 \%$ in localized cancer, $70 \%$ in regional metastasis, and $14 \%$ in distant metastasis. $^{16}$

Rectal cancer treatment depends on the tumor stage. Whether or not to take neoadjuvant therapy depends on tumor depth infiltration and lymph node metastasis. Therefore, preoperative evaluation of rectal cancer is very important in the selection of treatment and estimating the prognosis. There are two main forms of treatment for rectum cancers. While total mesorectal excision is sufficient in the early stages (T1-T2), neoadjuvant chemoradiotherapy is required in locally advanced stages $(\mathrm{T} 3 \mathrm{~cd}$, T4, or nodal metastasis). The most important imaging method for staging is MRI. MRI evaluates the rectum wall anatomical layers, perirectal area, mesorectal fascia, and lymph nodes very well. The most accurate evaluation of MRF invasion and circumferential resection margins is done with phase array MRI. ${ }^{17}$ In addition, MRI is the best method to demonstrate the spread of rectum cancer to the pelvic organs (prostate gland, uterus, vagina, and perianal muscles).

In the literature, with $1.5 \mathrm{~T}$ MRI, $\mathrm{T}$ staging accuracy rates range from $67-88 \% .^{18,19,20}$ Staging accuracy rate of our study was $72.2 \%$ and was compatible with the literature. Nine of the $12 \mathrm{~T} 2$ stage patients (75\%) were staged correctly by MRI. The remaining 3 patients (25\%) were staged as T3. High staging in T2 stage cancers is one of the defects of MRI. Inflammatory-desmoplastic changes surrounding the tumor, fibrous tissue and hypervascularity are 


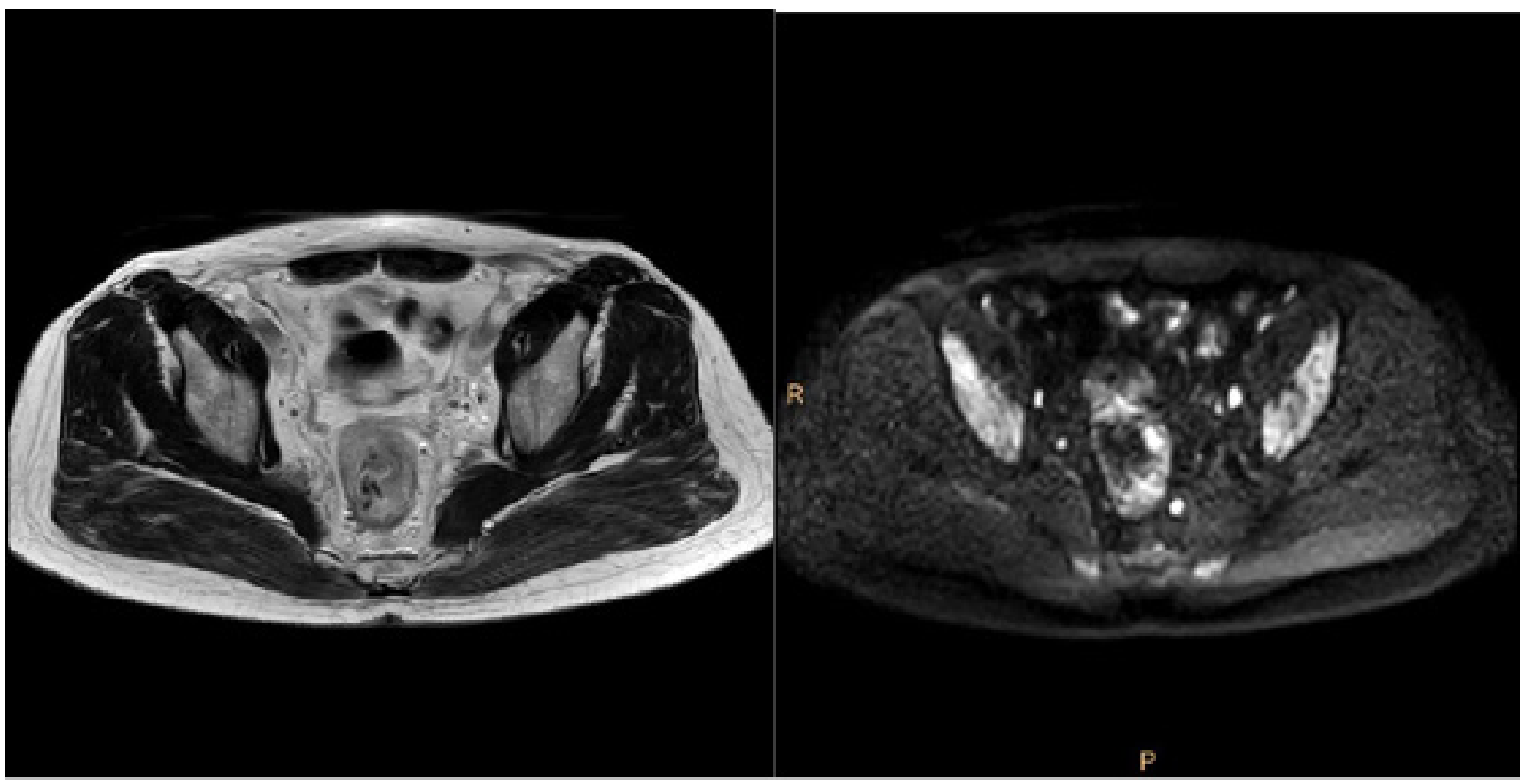

Figure 3. 63 years old, male patient. Histopathology result: pT3NO. According to MRG, perirectal adipose tissue extension of the tumor and DWI hyperintense lymph node (mT3 N1).

factors that cause high staging on MRI. These factors affect the muscularis propria layer and make it difficult to differentiate tumor borders. Tumor localization is another factor for mis-staging. ${ }^{17,19}$, ${ }^{21}$ Nine of 12 T3 stage patients (75\%) were correctly staged with MRI. The remaining 3 patients (25\%) were staged as T2. The separation of T2 and T3 stage rectal cancers is made according to the perirectal adipose tissue extension. However, it is difficult to distinguish between the $\mathrm{T} 2$ stage and early stage $\mathrm{T} 3$ tumors and this is an important diagnostic problem of MRI. ${ }^{21}$ Tumors with microscopic invasion into the perirectal adipose tissue are less staged with MRI. ${ }^{19}$ In our study, early-stage T3 patients were staged as T2 with MRI. But T3 stage tumor ADC values were found significantly lower than T2. Therefore, it was thought that adding DWI and ADC to conventional MRI will increase the T staging accuracy rate and contribute to T2-T3 separation. In addition, the prognosis of extramurally located tumors (T3-T4) is worse than murally located tumors (T1-T2). In our study, ADC values of extramurally located tumors (T3-T4) were significantly lower than murally located $\mathrm{T} 2$ tumors. This finding shows that ADC will help about the mural and extramural location of the tumor.
$\mathrm{N}$ staging accuracy rate of MRI in the literature ranges between $43-85 \%{ }^{23,24}$ The accuracy rate we found with T2 and DWI sequences was consistent with the literature. Diffusion creates image contrast with cellular density differences between tissues. ${ }^{25}$ Lymphoid tissue having high cellular density causes restriction in diffusion and hyperintense appears on DWI. This facilitates the detection of lymph nodes. Diffusion has been shown to be a valuable technique in showing lymph nodes. ${ }^{26}$ In one study, the total number of lymph nodes detected on DWI was found to be $6 \%$ higher than the number of lymph nodes detected by the T2 sequence. ${ }^{27}$ In the same study, no significant difference was observed between benign and metastatic lymph nodes in the visual evaluation of lymph nodes by diffusion. ${ }^{27}$ According to our findings, diffusion makes mistakes by performing top staging in $\mathrm{N}$ staging. This is due to the fact that benign lymph nodes appear hyperintense on DWI. ADC value can be helpful to reduce this error (Figure 3, 4).

Tumor-invasive lymph nodes have a histopathological organization of the primary tumor. Therefore, metastatic lymph nodes are expected to show similar diffusion with the tumor. For this reason, studies were performed with lymph node ADC and 


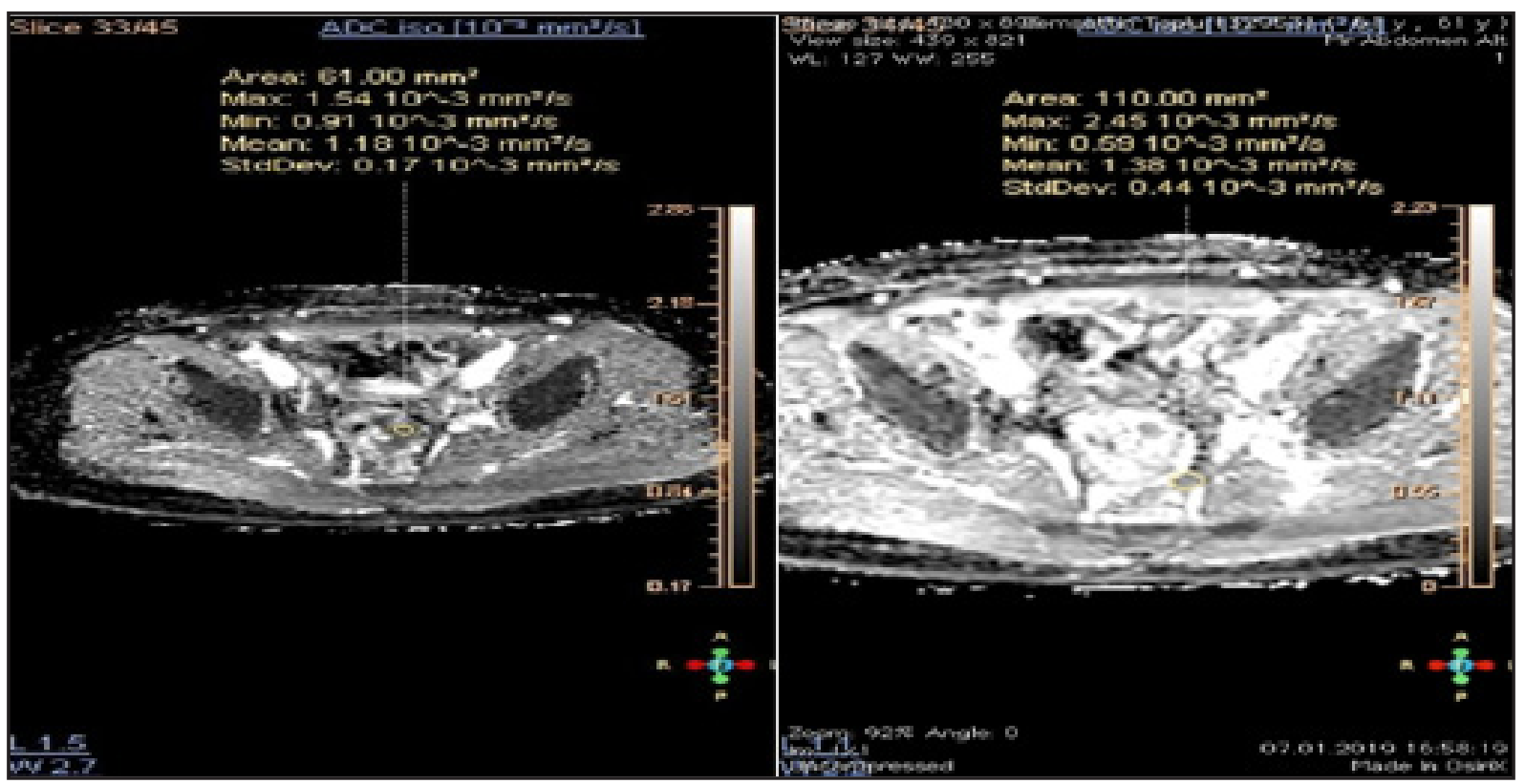

Figure 4. The tumor wall ADC value of the same patient was $0.91 \times 10^{-3} \mathrm{~mm}^{2} / \mathrm{sn}$ and was compatible with the extramural ADC value. The ADC value of the DWI hyperintense lymph node was above the lymph node cut-off value with $1.38 \times 10^{-3} \mathrm{~mm}^{2} / \mathrm{sn}$. According to DWI, the patient was N1. But according to ADC cut-off value, it was NO compatible with histopathology.

relative ADC values. In a study with $1.5 \mathrm{~T}$ MRI, it was found that ADC and relative ADC values of metastatic lymph nodes decreased significantly compared to benign lymph nodes. According to the relative lymph node ADC cut-off value, the lymph node staging the accuracy rate was 78.5. According to the lymph node ADC cut-off value, the accuracy rate was 74.8 . In the same study, according to the size criteria, the lymph node staging accuracy rate was found $62 \% .{ }^{28}$ Highest accuracy rate was obtained with relative ADC values ${ }^{28}$ In our study, according to ADC cut-off values, the accuracy rate of lymph node staging was $83 \%$. This value was higher than the accuracy rate of lymph node staging with $\mathrm{T} 2$ and DWI sequence.

Although there are 62 metastatic lymph nodes histopathologically, ADC measurements were made from 33 lymph nodes most likely to be malignant, $5 \mathrm{~mm}$ and above. This shows that under $5 \mathrm{~mm}$, micrometastases are also accompanied. In addition, 18 lymph nodes that $5 \mathrm{~mm}$ and above were detected in $18 \mathrm{~N} 0$ patients. These findings show that the size criterion alone will not be reliable in $\mathrm{N}$ staging. ${ }^{29}$

According to the findings of our study, patients receiving neoadjuvant treatment had lower $\mathrm{T}$ and $\mathrm{N}$

UHOD

Number: 4 Volume: 30 Year: 2020 staging accuracy rates with MRI. Edema, inflammation, and necrosis caused by chemoradiotherapy may cause errors in staging with MRI. ${ }^{20}$

According to the histopathology results, no significant difference was found between the primary tumor ADC values of 18 cases with lymph node metastasis and the tumor ADC values of 18 cases with NO. In another study, it was seen that there was no significant correlation between tumor ADC value and $\mathrm{N}$ stage and it was compatible with our finding. ${ }^{30}$

Studies have shown that the pathological complete response correlates with increased ADC after chemoradiotherapy. ${ }^{22}$ In our study, primary tumor ADC values increased after neoadjuvant therapy and tumor size decreased in all patients. In tumors that respond well to neoadjuvant therapy, the ADC value increase was found significantly higher than the partially responding group. This finding showed that ADC can help predict neoadjuvant therapy efficacy Figure 5).

In our study, ADC values of poorly differentiated tumors were found to be significantly decreased compared with moderately and well-differentiated tumors. In another study, it was similarly shown that there is a significant correlation between ADC 


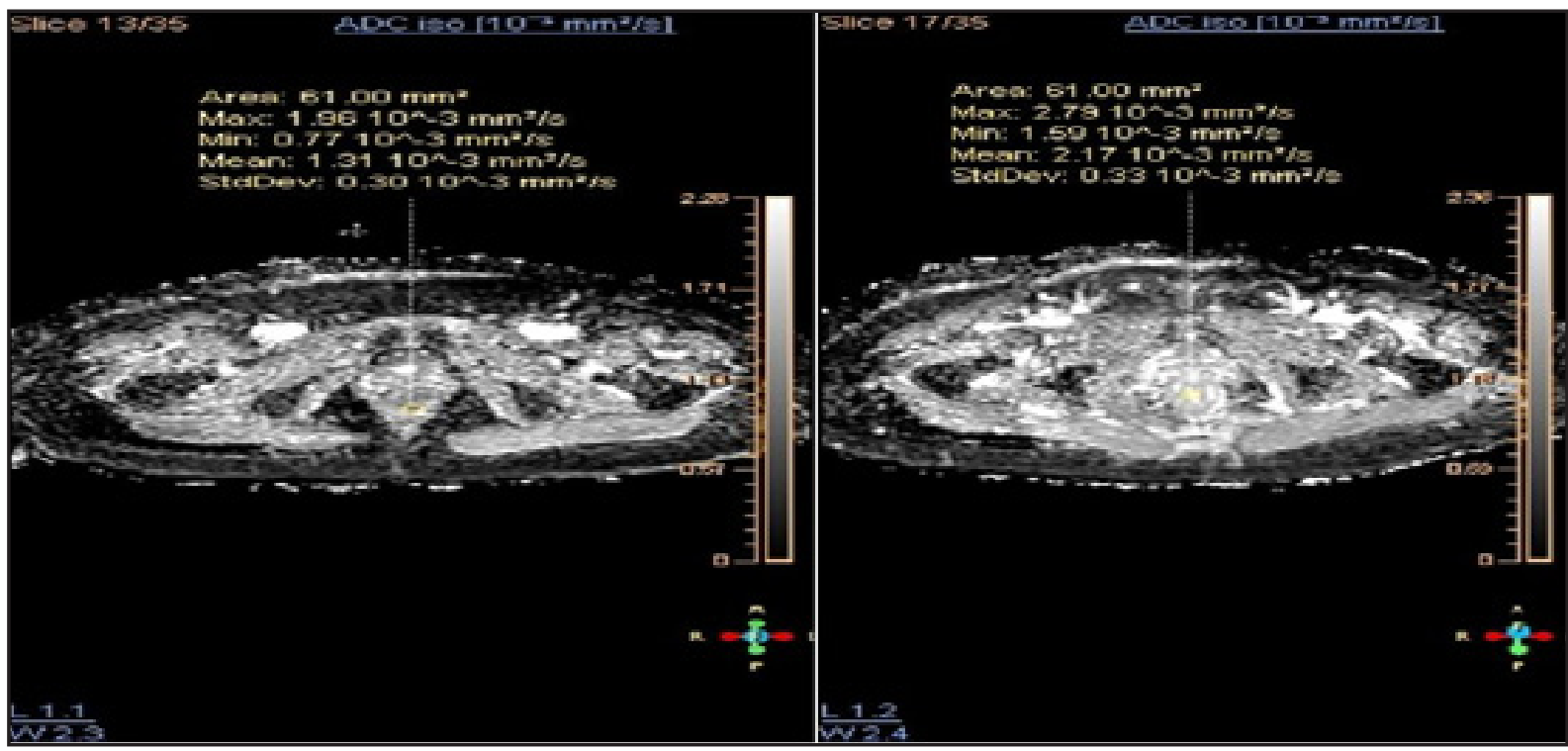

Figure 5. 68 years old, T2N1, patient with a good response to neoadjuvant therapy (Mandard TRG 2). Pretreatment ADC value was $1.31 \times 10^{-3} \mathrm{~mm}^{2} / \mathrm{sn}$, post treatment $\mathrm{ADC}$ value was $2.17 \times 10^{-3} \mathrm{~mm}^{2} / \mathrm{sn}$. $\Delta$ ADC was $0.8 \times 10^{-3} \mathrm{~mm}^{2} / \mathrm{sn}$ in accordance with good response. Pretreatment ADC value was appropriate with intramural location (T2).

value and tumor differentiation and ADC is a noninvasive potential marker showing tumor aggressiveness. ${ }^{9}$

Considering all findings, primary tumor ADC value helps to predict tumor mural-extramural location, differentiation grade, and response to neoadjuvant therapy. In addition, lymph node ADC values increase the accuracy rate in $\mathrm{N}$ staging. As a result, rectum tumor staging should be done according to conventional MRI and DWI sequences. ADC maps of patients should be obtained.

\section{REFERENCES}

1. Ferlay J, Soerjomataram I, Rajesh D, et al. Cancer fact sheet:GLOBOCAN 2012, Cancer Incidence and Mortality Worldwide. International Agency for Research on Cancer. 136: E359-386, 2015.

2. Wietek BM, Kratt T. Current MRI staging of rectal cancer. Rofo 184: 992-1001, 2012

3. Sagar PM, Pemberton JH. Surgical management of locally recurrent rectal cancer. Br J Surg 83: 293-304, 1996.

4. Pfister $\mathrm{K}$, Peleikis $\mathrm{H}$, Fürst $\mathrm{A}$, et al. Adjuvant radiochemistry. Does its use after total mesorectal excision improve the rates of local recurrence and/or the survival rates in patients with rectal carcinoma? Chir Prax 62: 373-381, 2004.
5. Breugom AJ, Van Gijn W, Muller EW, et al. Adjuvant chemotherapy for rectal cancer patients treated with preoperative (chemo)radiotherapy and total mesorectal excision: A Dutch Colorectal Cancer Group (DCCG) randomized phase III trial. Ann Oncol 26: 696-701, 2015.

6. Janjan NA, Crane C, Feig BW, et al. Improved overall surviva among responders to preoperative chemoradiation for locally advanced rectal cancer. Am J Clin Oncol Cancer Clin Trials 24: 107-112, 2001.

7. Glynne-Jones R, Wyrwicz L, Tiret E, et al. Erratum: Recta cancer: ESMO Clinical Practice Guidelines for diagnosis, treatment and follow-up. Ann Oncol 28: iv22-iv40, 2017.

8. Koh DM, Collins DJ. Diffusion-weighted MRI in the body: applications and challenges in oncology. AJR Am J Roentgenol 188: 1622-1635, 2007.

9. Curvo-Semedo L, Lambregts DMJ, Maas M, et al. Diffusionweighted MRI in rectal cancer: Apparent diffusion coefficient as a potential noninvasive marker of tumor aggressiveness. $J$ Magn Reson Imaging 35: 1365-1371, 2012.

10. Ross BD, Moffat BA, Lawrence TS, et al. Evaluation of cancer therapy using diffusion magnetic resonance imaging. Mol Cancer Ther 2: 581-587, 2003.

11. Edge SB, Byrd DR, Compton CC, Fritz AG, Greene FL, Trott A, editors. AJCC cancer staging manual (7th ed). New York, NY: Springer, 2010: 143-145.

12. Mandard AM, Dalibard F, Mandard JC, et al. Pathologic assessment of tumor regression after preoperative chemoradiotherapy of esophageal carcinoma. Clinicopathologic correlations. Cancer 73: 2680-2686, 1994. 
13. Kuipers EJ, Grady WM, Lieberman D, et al. Colorectal cancer. Nat Rev Dis Prim 1: 15065-15085, 2015.

14. Jhaveri KS, Hosseini-Nik H. MRI of Rectal Cancer: An Overview and Update on Recent Advances. AJR Am J Roentgenol 205: 42-55, 2015.

15. Gross CP, MCAvay GJ, Krumholz HM, et al. The effect of age and chronic illness on life expectancy after a diagnosis of colorectal cancer: implications for screening. Ann Intern Med 145: 646-653, 2006.

16. Siegel RL, Miller KD, Fedewa SA, et al. Colorectal cancer statistics, 2017. CA Cancer J Clin 67: 177-193, 2017.

17. Beets-Tan R, Beets $G$, Vliegen R, et al. Accuracy of magnetic resonance imaging in prediction of tumour-free resection margin in rectal cancer surgery. Lancet 357: 497-504, 2001.

18. Poon FW, McDonald A, Anderson JH, et al. Accuracy of thin section magnetic resonance using phased-array pelvic coil in predicting the T-staging of rectal cancer. Eur J Radiol 53: 256-262, 2005.

19. Akasu T, linuma G, Fujita $T$, et al. Thin-Section MRI with a Phased-Array Coil for Preoperative Evaluation of Pelvic Anatomy and Tumor Extent in Patients with Rectal Cancer. Am J Roentgenol 184: 531-538, 2005.

20. Giusti S, Buccianti P, Castagna M, et al. Preoperative rectal cancer staging with phased-array MR. Radiat Oncol 7: 2939, 2012.

21. Brown G, Richards CJ, Newcombe RG, et al. Rectal Carcinoma: Thin-Section MR Imaging for Staging in 28 Patients. Radiology 211: 215-222, 1999.

22. Zhu HB, Zhang XY, Zhou XH, et al. Assessment of pathological complete response to preoperative chemoradiotherapy by means of multiple mathematical models of diffusion-weighted MRI in locally advanced rectal cancer: A prospective singlecenter study. J Magn Reson Imaging 46: 175-183, 2017.

23. Ferri M, Laghi A, Mingazzini $P$, et al. Pre-operative assessment of extramural invasion and sphincteral involvement in rectal cancer by magnetic resonance imaging with phasedarray coil. Color Dis 7: 387-393, 2005.

24. Kim JH, Beets GL, Kim MJ, et al. High-resolution MR imaging for nodal staging in rectal cancer: are there any criteria in addition to the size? Eur J Radiol 52: 78-83, 2004.
25. Kwee TC, Takahara T, Ochiai R, et al. Diffusion-weighted whole-body imaging with background body signal suppression (DWIBS): features and potential applications in oncology. Eur Radiol 318: 1937-1952, 2008.

26. Nakai G, Matsuki M, Inada $Y$, et al. Detection and Evaluation of Pelvic Lymph Nodes in Patients With Gynecologic Malignancies Using Body Diffusion-Weighted Magnetic Resonance Imaging. J Comput Assist Tomogr 32: 764-768, 2008.

27. Heijnen LA, Lambregts DMJ, Mondal D, et al. Diffusionweighted MR imaging in primary rectal cancer staging demonstrates but does not characterise lymph nodes. Eur Radiol 23: 3354-3360, 2013.

28. Yasui $O$, Sato M, Kamada A. Diffusion-weighted imaging in the detection of lymph node metastasis in colorectal cancer. Tohoku J Exp Med 218: 177-183, 2009.

29. Mönig SP, Baldus SE, Zirbes TK. Lymph node size and metastatic infiltration in colon cancer. Ann Surg Oncol 6: 579-581, 1999.

30. Akashi M, Nakahusa Y, Yakabe T, et al. Assessment of aggressiveness of rectal cancer using 3-T MRI: correlation between the apparent diffusion coefficient as a potential imaging biomarker and histologic prognostic factors. Acta radiol 55: 524-531, 2014.

\section{Correspondence:}

Dr. Mustafa YILDIRIM

Elazig Fethi Sekin Sehir Hastanesi

Radyoloji Bölümü

23100 Dogukent

23100, ELAZIG / TURKEY

Tel: (+90-424) 6066000

e-mail: mustafa23468@outlook.com

\section{ORCIDs:}

Mustafa YILDIRIM

0000-0001-6874-9294

Mustafa KOC 